\title{
Challenges for Smart Environments in Bathroom Contexts
}

\author{
Christian Leichsenring \\ cleichsenring@techfak.uni- \\ bielefeld.de \\ Jan Hammerschmidt \\ jhammers@techfak.uni- \\ bielefeld.de
}

\author{
Jiajun Yang \\ jyang@techfak.uni- \\ bielefeld.de \\ Thomas Hermann \\ thermann@techfak.uni- \\ bielefeld.de
}

\author{
Center of Excellence Cognitive Interaction Technology (CITEC) \\ Ambient Intelligence Group \\ Bielefeld University \\ Bielefeld, Germany
}

\begin{abstract}
Smart homes have been mostly treated as homogeneous environments where each room is distinguished by the activities performed there but not by any fundamentally different basic parameters for systems to operate in. We argue that at least for bathroom environments, things like the extensive presence of liquid water and humidity and special privacy considerations challenge these assumptions. We discuss typical and unique challenges for ubiquitous computing interfaces in bathroom environments and we look at how actual and conceptual systems confront these challenges. We review bathroom systems in the literature and present two systems of our own to exemplify the unique challenges to smart environments the bathroom provides, one of which is presented here for the first time.
\end{abstract}

\section{CCS Concepts}

-Human-centered computing $\rightarrow$ Sound-based input / output; Haptic devices; Auditory feedback; Activity centered design; Scenario-based design; Contextual design; Ubiquitous and mobile computing systems and tools;

\section{Keywords}

Smart Home; Smart Environments; Tangible Interaction; Sonification; Ambient Intelligence; Soundscape

\section{INTRODUCTION}

Smart environments are enjoying increasing popularity both in research as well as in actual applications in people's homes. However, smart homes have been mostly treated as largely homogeneous environments in that each room is

Permission to make digital or hard copies of all or part of this work for personal or classroom use is granted without fee provided that copies are not made or distributed for profit or commercial advantage and that copies bear this notice and the full citation on the first page. Copyrights for components of this work owned by others than ACM must be honored. Abstracting with credit is permitted. To copy otherwise, or republish, to post on servers or to redistribute to lists, requires prior specific permission and/or a fee. Request permissions from permissions@acm.org.

ICMI 2016 Workshop on Embodied Interaction with Smart Environments November 12, 2016, Tokyo, Japan

(C) 2016 ACM. ISBN 123-4567-24-567/08/06 .. \$15.00

DOI: $10.475 / 123 \_4$ maybe distinguished by the activities performed there but not by substantially different basic parameters in which systems have to operate. Bathrooms have not only been neglected in the literature so far, even systems that are applicable in the bathroom sometimes fail to address the specific environment found there. One notable exception is Lashina [13] who took an early focused look at smart bathroom environments and brought up design considerations over wet hands, losing remote controls and noise sources.

In this paper, we discuss the challenges we see as unique to or at least typical for bathroom environments and we then proceed to look at how actual systems confront these challenges and finally how more speculative systems could confront them. To this end, we take a look at how bathroom systems were handled in the literature so far, whether other researchers faced the same challenges we identified and how they tackled them. We then present two systems we developed ourselves, one being a novel system that produces multimodal ambiances and that is controlled by a tangible user interface especially suited for bathroom environments, the other being an assistance system to reduce the energy consumption of showering and that was first presented by Hammerschmidt et al. [8]. We discuss how they relate to our framework for bathroom environments and finally, we discuss some avenues for future research that could be taken to address the challenges we outline in novel ways and we argue that investigating bathroom environments in more depth is necessary.

\section{SPECIAL CHALLENGES IN BATHROOM ENVIRONMENTS}

While in many aspects, designing systems for smart bathrooms is no different from designing any other smart home system, there are some conditions and challenges that are specific to bathrooms.

C1 Humidity is a major concern, since it can lead to condensation in electronics, on screens, capacitive surfaces and sensors, and even reduce the visibility in the room itself when fogging occurs.

C2 Similarly, liquid water in the form of splashes, jets or even full immersion is a related concern. It requires 
special protection of electronics and wiring, wet fingers make touch screens more difficult to use and even tangible interaction can be affected by slippery fingers or materials that do not react well to moisture.

C3 Privacy concerns might play a bigger role for many users in the bathroom than they do for other rooms such as the kitchen or the living room. In our own laboratory apartment [10], we abstained from using any cameras in the bathroom and some might even be more comfortable if microphones or other forms of sensors were banned from the bathroom as well.

C4 If microphones are used, the acoustic environment in a bathroom tends to be more challenging than usual, with its strong reverberation and echo and the broadband noise water can produce. The same characteristics can also interfere with producing sound, e.g. when an audio signal is masked by the sound of running water. Conventional workarounds in noisy environments such as beamforming might not work as well as in other places, given the source of noise is usually close to the user and also due to the especially echoey quality of bathrooms.

C5 Besides affecting the acoustics, the usually confined space of a bathroom has the additional effect of restricting the number and volume of any components introduced, especially when unobtrusiveness and aesthetic considerations are a concern; every piece of technology or anything else that stands out in an undesirable way might be more noticeable than it would be in a larger room. This lack of space is especially challenging for smart environments that include some kind of physical agent that needs to navigate these confined surroundings and might even be required to do so with restricted sensory capabilities as per C3. Lashina [13] also adds to this that confined spaces tend to get cluttered, so having small devices or remote controls to control a system might not be a good idea as they might be hard to find when needed.

C6 Wearables share the requirements regarding water resistance mentioned in $\mathrm{C} 2$ but some, especially smart clothing, have the additional issue of simply not being worn in the bathroom and therefore not being available for data collection.

C7 While glasses might simply be called the oldest piece of wearable technology and therefore subsumed under C6, since they are still by far the most common, they deserve their own mention. Especially when taking a shower, the bathroom is oftentimes a place where people who usually depend on them will take off their glasses. In addition to the technical difficulties discussed in $\mathrm{C} 1$ and $\mathrm{C} 2$, this severely limits the design space of visual displays, as it makes using too complex or small interfaces highly problematic.

We will refer back to these challenges when discussing concrete systems in the following sections.

However, another consideration specific to bathrooms are the data sources that are available and that inform specific use cases. These comprise
- energy consumption, especially for heating water but also for hair dryers and other electrical heating appliances,

- water consumption,

- usage of bathroom products such as shampoo, lotions and soap,

- water and air temperature,

- health information, including weight and BMI but also more advanced sources of health-related information as toilets that are already in widespread use in some areas provide, and

- news and entertainment media that might be an important way to complement a maybe less interesting primary task with a secondary task that makes the overall experience more enjoyable and might give the users the feeling of increased productivity for the time spent in the bathroom.

\section{BATHROOM SYSTEMS IN THE LITER- ATURE}

Smart mirrors are particularly attractive in bathroom context because, it is usually the most visually salient object in a bathroom and one of the objects that users interact with the most. Lashina [13] investigated smart bathrooms and besides identifying some of the same challenges we did (cf. Section 2), she implemented a bathroom mirror that helps to reduce clutter and the number of visible devices by using an object that is already present in virtually all bathrooms (cf. C5). She then adapted the mirror further to special bathroom needs by removing the need to actually touch the glass surface to avoid problems with wet fingers and fingerprints in general (C2) and added a touch-sensitive frame for the same reasons. Generally, smart mirrors currently enjoy quite a bit of attention by academic researchers and industry alike (e.g. Braun [4] and Hossain et al. [11]).

Smart faucets similarly are a common theme in smart homes, including in the bathroom. Bonanni et al. [2] for example used colored light and audio-visual feedback to indicate water temperature aiming at conserving water.

Chen et al. [5] used acoustic means for person tracking in bathrooms to address the privacy concerns with cameras there (cf. C3).

Bathonify is an interactive bathtub soundscape system developed by Hirai [9] that uses information primarily from patterns detected from the water pressure sensor as well as electrocardiography (ECG) sensor to the user. Different types of human interaction with the water such as stirring and going in and out of the tub are detected and used as control parameters. Although the idea is certainly interesting, the choice of ECG might be of limited practicality in real life scenarios (C6), especially in relation to the moist bathroom environment (C1 and $\mathrm{C} 2)$.

Koike et al. [12] developed a projection-based display and interactive system to project visual information onto the water surface of a bathtub. The system uses a projector as its display and a Mircosoft Kinect to obtain depth information. This system does not try to work around the bathroom environment but instead uses the unique circumstance of a user being immersed in a body of water to its advantage. Various 


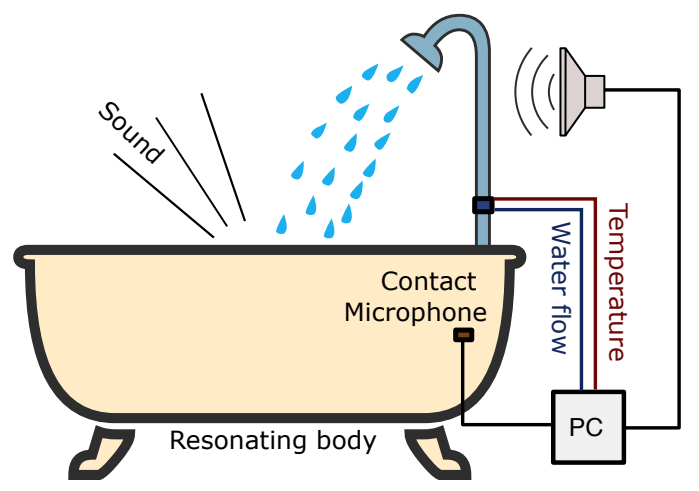

Figure 1: Hardware setup of the Sonic Shower system: Based on the current water flow and temperature data, the sound that is transmitted via the resonating body of the bathtub and picked up by the contact microphone is processed and, through a speaker, directly fed back to the user. Note that in our current setup, we use a shower tub with the microphone installed on its underside, which works just as well. [8]

modes of physical interaction can be used to interact with GUI elements, such as stroking and scooping the water and especially poking through the water surface from below, a mode of interaction that to our knowledge no other interface even physically allows. The use of Kinect RGB-D cameras provides a suitable way to obtain the needed information. However, the device might possibly suffer from the usual $\mathrm{C} 1$ and $\mathrm{C} 2$ issues but most importantly, with an RGB camera pointing straight down at an actively used bathtub, privacy concerns are especially pressing (C3). A production system might address these concerns by for example requiring a hardware component that is electronically unable to output anything other than the depth information, which is presumably less useful to an attacker. This is not easy to achieve, however, as long as an RGB or grayscale image is used in obtaining the depth information at some point in the processing chain.

An interesting extension of the system by Koike et al. would be to include the foam of a bubble bath as an active control element that can be formed and sculpted in unique ways.

Based on these bathroom systems by other authors, in the next two sections, we present two interactive systems we installed in our laboratory environment, the Cognitive Service-Robotics Apartment (CSRA). This apartment is a smart home environment that has a kitchen, living room area, sports room and, most relevant to this paper, a fully functional bathroom that comprises a sink, a toilet and a shower. For an overview of the CSRA as a whole an the sensors and actors present, please refer to Holthaus et al. [10].

\section{INFODROPS - SONIC SHOWER}

As a first exemplary system, illustrating one possible way to deal with the challenges that we are faced with in bathroom environments, we want to present our InfoDrops system.

The InfoDrops system was primarily designed for users to receive feedback about their energy and water consumption when taking a shower [8]. Our main motivation for the work on this system was our observation that showering is not only an activity producing a rather "hidden" resource consumption, but also one that is more resource-intensive than most people think. In the United States, for example, domestic water heating accounts for between 15 and 25 percent of the energy consumed in homes [6]. Even unheated, water treatment itself makes a considerable contribution to greenhouse gas emissions [15]. Add to that the fact that taking a shower in the Western world usually uses up more water than the typical person living in a developing country uses in a whole day [17] and it becomes clear that a reduction of water consumption is both desirable and feasible.

\subsection{Design Process and Dealing with Individ- ual Challenges}

When designing the InfoDrops system, we very soon decided to give users immediate feedback (as opposed to, for example, giving a post-hoc analysis or only general information), as it has been shown that this is the most effective way for changing consumption habits [7]. In consequence, this forced us to deal with the challenges specific to bathroom environments, as outlined in Section 2.

\subsubsection{Display Design}

Of all activities that can take place in a bathroom, taking a shower is probably the most difficult one to address when thinking about smart environments:

- Wearers of glasses will most certainly take them off during a shower $(\mathrm{C} 7)$, restricting the possibilities for a visual interface to relatively simple visualizations.

- Furthermore, putting one's head in a spray of water, as it is usually done when showering, not only forces most people to close their eyes and therefore interrupt any possibility to interact or even attend to a visual display, but usually leaves the vision impaired for a longer period of time.

- Any auditory display would have to work against the broadband noise of running water (C4).

- Finally, a shower (or bathtub) is the bathroom's area that is most heavily exposed to humidity (C1) and water $(\mathrm{C} 2)$, posing a challenge for any electronics that would be installed there.

Considering those difficulties, which would mostly affect visual displays, we decided to instead use an auditory one, as this resolves any problems with restricted vision and make installing electronic components (or even the interface itself) in the shower area unnecessary (a loudspeaker can be placed at a safe distance).

This of course leaves us open to C4. Our solution, that we present in the following, is to modify the already existing sounds instead of overlaying new ones onto the soundscape that could mask them.

\subsubsection{Using Blended Sonification}

While using an auditory display obviously reduces the number of difficulties specific to bathroom environments, it comes with some challenges of its own that need to be considered. First, any auditory display that is meant to be a casual interface (as opposed to, for example, a sonification 


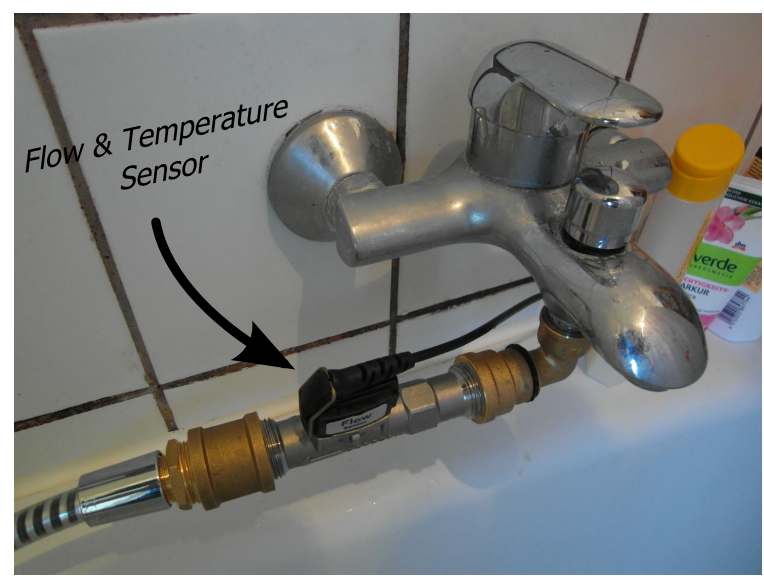

Figure 2: Picture of the flow sensor used in the InfoDrops setup.

that is used in a professional context) has a high risk of being perceived as annoying, since the user is constantly exposed to it and contrary to a visual display, you obviously cannot just 'look away'. Also, the sounds produced by forcing water through the nozzles of a shower head and those produced by water drops falling onto the tub surface can very easily mask (or at least interfere with) any additional sound produced by an auditory display (also cf. C4).

One possible way to deal with this problem would be to analyze the frequency spectrum of the water noise and generate sounds that are outside of this spectrum. However, due to the broad-band nature of the signal and the comparatively high volume of the water noise, this would make the use of a relatively loud and high-pitched sounds necessary, which inevitably would lead to the auditory display being perceived as highly annoying.

Instead, we decided to work with the existing soundscape as much as possible, as has been proposed in the framework of Blended Sonification by Tünnermann et al. [16], a conceptual extension of the approach of Auditory Augmentation by Bovermann et al. [3]. The basic setup to achieve this is shown in Figure 1: a contact microphone captures the sound of water falling onto the shower tub and feeds this signal into the processing unit as input for the sonification. Depending on the consumption values captured by a flow sensor (cf. Figure 2), the sound is filtered, modified and, through a speaker, directly fed back to the user, so that, together with the water noise, it appears as a coherent sound unit. Note that using a contact microphone also limits privacy issues since it does not usually capture any intelligible speech (C3). An exemplary sound example of the InfoDrops system can be found at https: //pub.uni-bielefeld.de/download/2906095/2906096 [14].

\section{MULTIMODAL AMBIANCES}

For the second interface for bathroom environments, we present an audiovisual ambient system installed in the bathroom of the CSRA. Our objective is to create immersive audiovisual scenarios by combining ambient soundscapes and mood lighting to create emotive and enjoyable atmospheres. This system uses small tangible objects as the control medium to select different themes.

The remainder of this section explains the system in three parts: physical control, soundscape and lighting design.

\subsection{Tangible Control Design}

For this project, we first narrowed the design criteria for the tangible control interface to the following:

- We would like to use tangible objects to trigger the ambiances rather than using a screen-based interface or the conventional remote control style. Tangible interaction is arguably one of the styles of interaction the least affected by many of the challenges outlined in Section 2.

- The use of electronic components should be minimized due to humidity concern (C1). All electronic components should be well sealed to prevent water damage (C2).

- From an aesthetic point of view, the tangible objects should not only be able to function as a controller but also serve as a common home decoration.

Based on these points, we finalized the design shown in Figure 3. The object with the black top is the wooden dock (receiver) with an RFID reader inside. The dock is connected to an Intel NUC in the bathroom ceiling via USB. That computer is connected to the rest of the CSRA via gigabit Ethernet. The dock is painted with water resistant coating.

The five objects symbolize one ambiance each. The shell represents the beach theme, the small cartoon owl represents the nighttime theme, the small piece of wood represents the forest theme, and the glass pebble represents a meditative theme with more abstract sounds, in that the soundscape is an ambient electronic music we composed rather than recreating the soundscape of a physical location. An additional "off" object is used to reset the bathroom ambiance to neutral white light and silence, effectively shutting off the system. Each object works by having an RFID tag attached to the bottom. All the tangible objects used for control have some extent of water resistance and are able to withstand the humidity levels of the bathroom.

The interface is placed on a little shelf space right above the basin. To use the device, the user can simply select a theme by choosing the object that symbolizes it and place it on the wooden dock. Once the object is placed on the dock, the computer will register the tag ID and trigger the according theme, controlling the sound software via Open Sound Control (OSC) and the lighting components via the

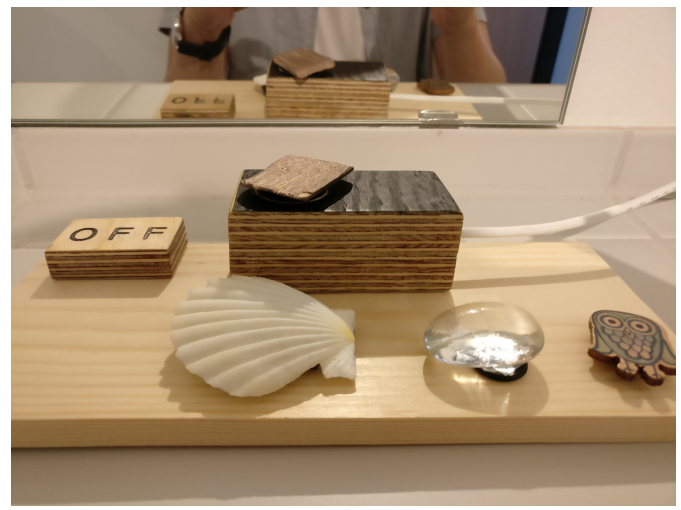

Figure 3: The tangible control objects for the ambiance control. The forest theme is selected in the picture. 
common CSRA middleware RSB [19]. The control script is written in Python and the soundscape is generated using SuperCollider.

\subsection{Soundscape Design}

The soundscape generated from this system is not a fixed predefined sound loop. Instead, we prepared a collection of sound samples, including sounds such as birdsong, ocean waves, and wind noises. Each sound file is labeled based on two categories: 1 . the theme it belongs and 2 . whether the sound is regarded as a background ambiance $(>10 \mathrm{~s})$ or a single object sound $(<10 \mathrm{~s})$. Taking the forest theme as an example, the background ambiance consists of elements such as soothing wind sounds and a small creek. These sounds are played back constantly in a loop, laying out the sonic foundation of the theme. Then the single objects (e.g. animal sounds) will appear sparsely with randomized parameters such as sample selections, sample playback gaps, and audio effects (i. e. reverberation). Therefore, re-triggering the same theme will always result in different sonic outcomes.

\subsection{Lighting Design}

Philips Hues ${ }^{1}$ were used as a drop-in replacement for conventional light bulbs that allow color and light intensity to be changed wirelessly according to the soundscape selected. The bathroom being a closed room with no windows actually worked to our advantage here because we had a much tighter control over both light color and light intensity than if we had to deal with an environment that is dominated by the color and intensity of the outside light. Figure 4 shows four of the lighting presets. These are (a) the baseline setting in a neutral white, (b) the beach atmosphere with maximum intensity light that is warm and yellow-orange at the ceiling to evoke sunlight and blue for the lower lights as to be reminiscent of the sea. Then there is (c) the warm orange but darker ambiance for the meditative theme, and (d) the forest theme with its light green light. All pictures were taken with identical exposure settings, so they represent absolute brightness, not the perceived brightness that will differ from the images especially for the dark ambiances due to the dark adaptation of the human eye. Not shown is the nighttime ambiance which has a blue tint and is of course also quite dark.

\subsection{Video Example}

A video example can be found at the following location: https://pub.uni-bielefeld.de/download/2906095/2906097 or as Leichsenring et al. [14].

\section{CONCLUSION}

\subsection{Summary}

In this paper, we raise seven challenges for designing smart system in bathroom contexts that are characteristic to bathroom conditions. The specific points we addressed were humidity and liquid water and their effect on hardware, the increased importance of privacy and how this restricts the choice of sensors that can be used, the special acoustic characteristic in bathrooms, the restrictions of the usually confined space, and the restriction on wearables and glasses as a result of typical bathroom activities.

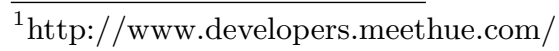

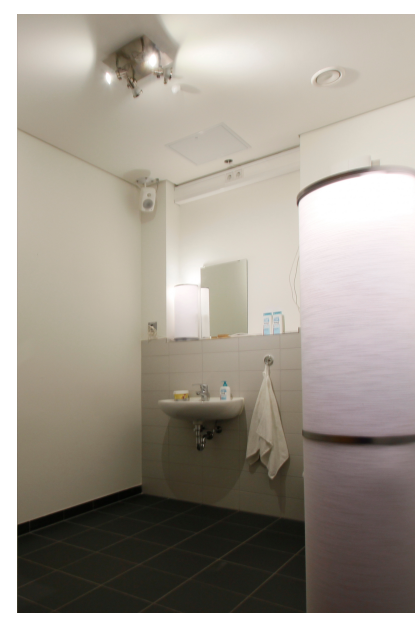

(a) Neutral white

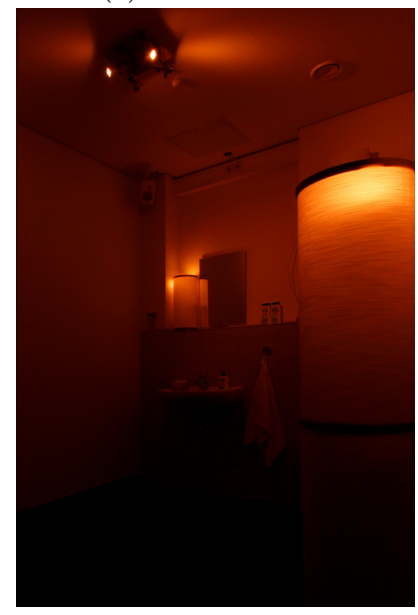

(c) Meditative theme

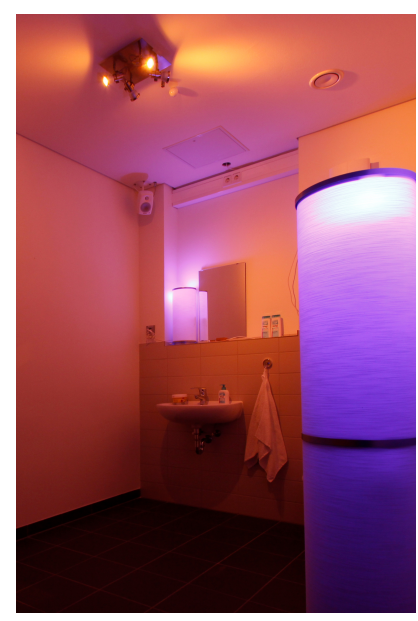

(b) Beach theme

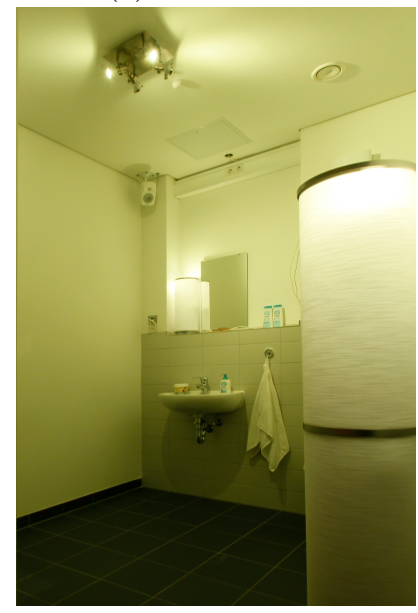

(d) Forest theme
Figure 4: Lighting presets for the bathroom ambiances.

Having looked at some implementations for smart bathroom systems, the most pressing issues that emerge seem to be related to water, privacy and space restrictions. We presented two bathroom system, where one focuses on providing information on enenrgy consumption during showering (InfoDrops) and the other focuses on providing relaxing audiovisual ambiances.

The InfoDrops system provides users with feedback about their resource consumption when taking a shower. It circumvents the problems that are introduced by humidity $(\mathrm{C} 1)$ and (running) water (C2) by employing an auditory display as its main means to interact with the user. This setup is exemplary for dealing with these challenges and, although obviously not usable as input, we can recommend using audio to convey information to the user when designing for a bathroom environment. Although the acoustics of such an environment can be challenging as well (C4), there are ways to deal with this, e.g. the approach of using Blended Sonification as we did for the Sonic Shower. To further improve the system, we plan to replace the current method of obtaining the resource consumption data via a flow sensor, which must be installed for the system to work. This is a more integrated approach, where the data is extracted from 
the information provided by a modern smart meter, working our way towards disappearing technology [18].

The multimodal ambiance installation allows users to easily trigger an immersive scene through the combination of environmental soundscape and colorful lighting. The use of tangible objects in this system is an example of tackling the challenges in $\mathrm{C} 1$ and $\mathrm{C} 2$. The physical objects are capable of withstanding the humidity and also water splashes because there is no electronic circuitry involved but only the RFID tag attached to the bottom. Compared to using touchscreen devices, especially when the user has wet hands, the tangible interface approach can be more reliable. From another standpoint, the control interface also can be regarded as a set of home decoration, which is efficient in a confined space environment (C5). To further improve the system, we plan to provide more means of interaction in order to give more precise results, e.g. the user could rotate or shake the tangible objects to give it certain parametric charge. Alternatively, the user could combine multiple objects. The system is also capable to be modified into a data driven system to provide ambient data display through soundscapes [20] and illumination. For instance, the room and water temperature can be mapped to a gradual variation of the ambiances from a cold to a hot climate.

\subsection{Outlook}

As mentioned in the introduction, bathrooms have been largely neglected so far, so we see a lot of different avenues future research might take and we hope having identified key challenges is a good guideline in that regard.

From the space constraint (C5) follows that existing surfaces are to be used whenever possible. This includes using them in a mixed-reality kind of fashion but this cannot happen using wearables as we have outlined, so projections are currently one of the better solutions we can see there. The washbasin for example is an excellent surface to project onto, being white, largely empty and right in front of the user during many tasks. Smart mirrors enjoy similar advantages, even though condensation might be a bigger problem for mirrors than for projectors. Tiles can also be used as projection surfaces or by embedding LEDs into them, as the material is somewhat translucent.

While water and condensation are certainly challenges for many forms of electronic devices, they could also be used in novel ways instead of being worked against. Both the water spray from a shower head as well as foggy air provide a volume of droplets that can be used for fog displays, 2D displays using projections and droplet timing [1] or even 3D volumetric displays.

On the other hand, bypassing the visual modality altogether with auditory displays seems like an even better way when possible, freeing the user from one specific surface and even more importantly, not needing any free surface area at all. Methods such as auditory display and speech recognition can satisfy such a requirement. However, there is the issue of the acoustic environment. We have shown one way to address this with Blended Sonification and there are surely others. If all else fails, adaptive sound level adjustment could always be applied.

Using technology, however, might also reduce clutter in bathrooms. The function of a traditional bathroom scale might for example be provided by special tiles or a smart bathroom rug.
Smart bathroom objects like a toothbrush might also be used to collect additional information, such as health-related data.

Beyond such ideas for concrete systems, there is also a lot of need for empirical and more basic research to pin down what the restrictions systems in bathrooms face really are. It is for example necessary to learn how different hardware sensors and actuators, especially those based on optical means, behave in real-life bathroom environments while they are being used. We hope with our capabilities in the CSRA, over time we will be able to provide some of these much needed answers.

\section{ACKNOWLEDGMENTS}

We would like to thank KatieAnna Wolf for her initial implementation and sound design work on the multimodal ambiances.

This work was supported by the Cluster of Excellence Cognitive Interaction Technology 'CITEC' (EXC 277) at Bielefeld University, which is funded by the German Research Foundation (DFG).

\section{References}

[1] Peter C. Barnum, Srinivasa G. Narasimhan, and Takeo Kanade. "A multi-layered display with water drops". In: ACM Transactions on Graphics 29.4 (2010), p. 1. ISSN: 07300301. DOI: 10.1145/1833351.1778813.

[2] Leonardo Bonanni, Ernesto Arroyo, Chia-hsun Lee, and Ted Selker. "Smart sinks: real-world opportunities for context-aware interaction". In: Conference on Human Factors in Computing Systems (2005), pp. 1232-1235. DOI: $10.1145 / 1056808.1056884$.

[3] Till Bovermann, Rene Tünnermann, and Thomas Hermann. "Auditory Augmentation". In: International Journal on Ambient Computing and Intelligence (IJACI) 2.2 (2010), pp. 27-41. DOI: 10.4018/jaci.2010040102.

[4] Max Braun. My Bathroom Mirror Is Smarter Than Yours. 2016.

[5] Jianfeng Chen, Jianmin Zhang, Alvin Harvey Kam, and Louis Shue. "An automatic acoustic bathroom monitoring system". In: Proceedings - IEEE International Symposium on Circuits and Systems (2005), pp. 1750-1753. ISSN: 02714310. DOI: 10.1109/ISCAS.2005.1464946.

[6] U.S. Department of Energy. Water Heating. [last accessed: 19.02.2013]. Aug. 2001. URL: http://apps1.eere. energy . gov / buildings / publications / pdfs / building america/26465.pdf.

[7] Corinna Fischer. "Feedback on household electricity consumption: a tool for saving energy?" In: Energy Efficiency 1.1 (May 2008), pp. 79-104. ISSN: 1570-646X. DOI: $10.1007 / \mathrm{s} 12053-008-9009-7$.

[8] Jan Hammerschmidt, René Tünnermann, and Thomas Hermann. "InfoDrops: Sonification for Enhanced Awareness of Resource Consumption in the Shower". In: Proceedings of the 19th International Conference on Auditory Display (ICAD-2013). Lodz, Poland, 2013. 
[9] Shigeyuki Hirai. "Emtertaining the Whole World". In: ed. by Adrian David Cheok, Teresa Ramão, Anton Nijholt, and Gino Yu. Springer London, 2014. Chap. Embedded Smarthouse Bathroom Entertainment Systems for Improvinv Quality of Life, pp. 85-114.

[10] Patrick Holthaus, Christian Leichsenring, Jasmin Bernotat, Viktor Richter, Marian Pohling, Birte Carlmeyer, Norman Köster, Sebastian Meyer zu Borgsen, René Zorn, Birte Schiffhauer, Kai Frederic Engelmann, Florian Lier, et al. "How to Address Smart Homes with a Social Robot? A Multi-modal Corpus of User Interactions with an Intelligent Environment". In: International Conference on Language Resources and Evaluation. European Language Resources Association (ELRA), 2016.

[11] M. Anwar Hossain, Pradeep K. Atrey, and Abdulmotaleb El Saddik. "Smart mirror for ambient home environment". In: Intelligent Environments (2007), pp. 589596.

[12] Hideki Koike, Yasushi Matoba, and Yoichi Takahashi. "AquaTop Display: Interactive Water Surface for Viewing and Manipulating Information in a Bathroom". In: Proceedings of the 2013 ACM international conference on Interactive tabletops and surfaces (2013), pp. 155164.

[13] Tatiana Lashina. "Intelligent bathroom". In: European Symposium on Ambient Intelligence (EUSAI 2004) 4.August (2004).
[14] Christian Leichsenring, Jiajun Yang, Jan Hammerschmidt, and Thomas Hermann. Challenges for Smart Environments in Bathroom Contexts - Video and Sound Example. DOI: http://doi.org/10.4119/unibi/2906095.

[15] Alina I. Racoviceanu, Bryan W. Karney, Christopher A. Kennedy, and Andrew F. Colombo. "Life-Cycle Energy Use and Greenhouse Gas Emissions Inventory for Water Treatment Systems". In: Journal of Infrastructure Systems 13.4 (2007), pp. 261-270. DOI: 10.1061/ (ASCE)1076-0342(2007)13:4(261).

[16] René Tünnermann, Jan Hammerschmidt, and Thomas Hermann. "Blended Sonification: Sonification for Casual Interaction". In: The 19th International Conference on Auditory Display (ICAD-2013). 2013.

[17] Kevin Watkins et al. "Human Development Report 2006 - Beyond scarcity: Power, poverty and the global water crisis". In: (2009).

[18] Mark Weiser and John S. Brown. "The coming age of calm technology". In: Xerox PARC 8 (1996), p. 2007.

[19] Johannes Wienke and Sebastian Wrede. "A middleware for collaborative research in experimental robotics". In: 2011 IEEE/SICE International Symposium on System Integration, SII 2011 (2011), pp. 1183-1190. DOI: 10. 1109/SII.2011.6147617.

[20] Katieanna E. Wolf, Genna Gliner, and Rebecca Fiebrink. "A Model for Data-Driven Sonification Using Soundscapes". In: Proceedings of the 20th International Conference on Intelligent User Interfaces Companion (2015), pp. 97-100. DOI: $10.1145 / 2732158.2732188$. 